\title{
Erythropoietin signaling increases neurogenesis and oligodendrogenesis of endogenous neural stem cells following spinal cord injury both in vivo and in vitro
}

\author{
HUI ZHANG ${ }^{1}$, XIAO FANG ${ }^{1}$, DAKE HUANG ${ }^{2}$, QINGLI LUO ${ }^{2}$, MEIJUAN ZHENG ${ }^{3}$, \\ KANGKANG WANG ${ }^{1}, \mathrm{LECAO}^{1}$ and ZONGSHENG YIN ${ }^{1}$
}

\author{
${ }^{1}$ Department of Orthopedics, The First Affiliated Hospital of Anhui Medical University, Hefei, Anhui 230022; \\ ${ }^{2}$ School of Basic Medical Science, Anhui Medical University, Hefei, Anhui 230032; ${ }^{3}$ Department of Clinical Laboratory, \\ The First Affiliated Hospital of Anhui Medical University, Hefei, Anhui 230022, P.R. China
}

Received May 15, 2017; Accepted October 13, 2017

DOI: $10.3892 / \mathrm{mmr} .2017 .7873$

\begin{abstract}
Erythropoietin (Epo) promotes functional recovery following spinal cord injury (SCI); however, the exact underlying mechanisms are yet to be determined. Although endogenous neural stem cells (NSCs) in the adult spinal cord are a therapeutic target in SCI models, the effect of Epo on this NSC population remains unknown. The present study investigated the effects of Epo on endogenous NSCs in the adult spinal cord both in vitro and in vivo. For the in vivo analyses, normal rats (Normal) and SCI contusion model rats (SCI) received either recombinant human Epo or saline treatment for 7 days $(5,000 \mathrm{U} / \mathrm{kg})$, and spinal cords were subsequently analyzed at 2,8, and 14 days. For in vitro analyses, NSCs harvested from adult rat spinal cords were exposed to Epo $(10 \mathrm{U} / \mathrm{ml})$. A significant increase in $\beta$-tubulin ${ }^{+}$new neurons $(\mathrm{P}<0.01)$ was observed at all three time points and $\mathrm{O}^{+}$new oligodendrocytes $(\mathrm{P}<0.05)$ at days 8 and 14 in the $\mathrm{SCI}+\mathrm{Epo}$ group compared with the SCI+Saline group. This was concomitant with a prolonged activation of Epo signaling; however, no effect on
\end{abstract}

Correspondence to: Dr Zongsheng Yin, Department of Orthopedics, The First Affiliated Hospital of Anhui Medical University, 218 Jixi Road, Hefei, Anhui 230022, P.R. China

E-mail: yinzongsheng@ahmu.edu.cn

Abbreviations: BBB, Basso, Beattie, and Bresnahan; BrdU, bromodeoxyuridine; BSCB, blood-spinal cord barrier; CNS, central nervous system; DAPI, 4',6'-diamidino-2-phenylindole; DMEM/F12, Dulbecco's modified Eagle's/F12 medium; Epo, erythropoietin; EpoR, erythropoietin receptor; FACS, fluorescence-activated cell sorting; FITC, fluorescein isothiocyanate; GFAP, glial fibrillary acidic protein; NSCs, neural stem cells; PBS, phosphate-buffered saline; rhEpo, recombinant human erythropoietin; SCI, spinal cord injury; SEM, standard error of the mean; TRITC, tetramethyl rhodamine isothiocyanate

Key words: erythropoietin, neural stem cells, neurogenesis, oligodendrogenesis, proliferation, spinal cord injury
NSCs proliferation was observed. Similar results were also obtained in vitro. Motor functional recovery was also noted at days 8 and 14 only in the Epo-treated SCI rats. Although the expression of Epo and EpoR significantly increased in Normal+Epo rats compared with Normal+Saline rats $(\mathrm{P}<0.05)$, the cell numbers and phenotype were comparable between the two groups. To the best of the author's knowledge, this is the first study to demonstrate that Epo signaling promotes both neurogenesis and oligodendrogenesis following SCI and that these may represent the underlying mechanisms for the functional recovery and therapeutic effects of Epo following SCI.

\section{Introduction}

Erythropoietin (Epo), a glycoprotein hormone (1), triggers erythroid progenitor cell proliferation and differentiation by binding to its specific membrane receptor (EpoR), which belongs to the type I family of single transmembrane cytokine receptors. Epo- and EpoR-null mice die in utero because of the lack of definitive erythropoiesis and mature erythrocytes (2). Both Epo and EpoR are expressed in the nervous system during development and adulthood (3), while Epo-null and EpoR-null mice exhibit thinning of the neuroepithelium and smaller brain size (4). Furthermore, Epo signaling supports neural cell survival during development (5), enhances neuronal recovery after injury in both the developing and mature brain (6), and enhances neurogenesis following brain injury $(5,7,8)$. The neuroprotective and neuroregenerative effects of Epo have been demonstrated in various central nervous system (CNS) diseases including ischemic stroke, schizophrenia, multiple sclerosis, brain injury, and spinal cord injury (SCI) (7). As a result, Epo has been used in clinical trials in patients with brain injury (7) and SCI (9). Under conditions of brain damage, the neuroprotective effects of Epo are mediated through EpoR-related signaling to stimulate neurogenesis (10).

Although the exact mechanisms of the neuroprotective effects of Epo are not fully understood, several processes have been implicated, including apoptosis inhibition, reduction of inflammation, restoration of vascular integrity, reduction of calcium influx, neurotoxic glutamate release, and reduction 
of lipid peroxidation by-products (9). Despite the well-documented Epo-induced recovery following SCI $(7,9,11)$, the effects of Epo on adult spinal cord neurogenesis have not been fully characterized. This may be due to the low rate of NSCs neuronal differentiation. In the adult spinal cord, endogenous NSCs proliferate following SCI (12), resulting strictly in a glial cell fate (13). Thus, the functional recovery remains less than satisfactory. Therefore, replacing injured or dead neurons and restoring signal transfer functionality are important processes in the NSC-related recovery following SCI. Newly born neurons following SCI have been observed only in cases of mild lesions, and this process is deemed abortive $(14,15)$. Thus, we speculated that Epo signaling may stimulate neurogenesis in endogenous NSCs following SCI. Here, we studied the effects of Epo treatment on the proliferation and differentiation of NSCs both in vitro and in vivo in the adult spinal cord under normal conditions and following SCI.

\section{Materials and methods}

Animals and SCI model. Specific pathogen-free adult female Sprague-Dawley rats (weight, 220-250 g; age, 6-8 weeks) were purchased from the Animal Center of Anhui, China. All experimental procedures were performed in accordance with the National Institutes of Health (NIH) Guide for the Care and Use of Laboratory Animals and were approved by the Institutional Animal Care and Use Committee of Anhui Medical University, (Hefei, China). The rats were housed under temperature-controlled conditions $\left(18-26^{\circ} \mathrm{C}\right)$ with a $12-\mathrm{h}$ light/dark cycle (lights $\mathrm{ON}$ at $6 \mathrm{AM}$ ) and ad libitum access to water and food. We adopted a modified version of Allen's method (16) to develop the rat contusion model of the T10 spinal cord. Briefly, after rats were anesthetized with pentobarbital sodium $(30 \mathrm{mg} / \mathrm{kg})$, a midline incision was made to uncover the spinal column at the T8-T11 level, followed by a T10 laminectomy to expose the spinal cord. To induce SCI, a $10-\mathrm{g}$ weight was dropped from a $2.5-\mathrm{cm}$ height onto the exposed and intact dura over the dorsal aspect of the spinal cord. Subsequently, the muscle, fascia, and skin were closed. Following the surgery, rats were administered subcutaneous saline and kept warm.

In vivo experimental procedures. The rats were randomly divided into four experimental groups ( $\mathrm{n}=18 \mathrm{each}$ ), consisting of two conditions (Normal or SCI) and two treatments over 7 consecutive days at the same time of the day (Saline or recombinant human Epo [rhEpo, 5,000 U/kg; Four Rings Biopharmaceutical Co. Ltd, Beijing, China)]. The Normal+Epo group consisted of normal rats treated with intraperitoneal (i.p.) injections of rhEpo over 7 consecutive days. The Normal+Saline group consisted of normal rats treated with i.p. saline at the same volume. The SCI+Epo group consisted of SCI rats treated with the i.p. rhEpo $(5,000 \mathrm{U} / \mathrm{kg})$ immediately after SCI onset over 7 consecutive days. The SCI+Saline group consisted of SCI rats treated with i.p. saline at the same volume.

For mitotic labeling, starting immediately after SCI onset, all rats received i.p. injections of bromodeoxyuridine (BrdU, 100 mg/kg; Thermo Fisher Scientific, Inc., MA, USA) once per day for 7 consecutive days. Rats were sacrificed 2, 8, or 14 days after SCI.
Cell isolation and culture. NSCs were isolated from the spinal cord of SCI rats $(n=3)$ as previously described (17) with minor modifications. Whole spinal cords were mechanically dissociated and cultured in Dulbecco's modified Eagle's/F12 medium (DMEM/F12; Invitrogen; Thermo Fisher Scientific, Inc., Waltham, MA, USA) supplemented with 2\% B-27 (Invitrogen; Thermo Fisher Scientific, Inc.), $20 \mathrm{ng} / \mathrm{ml}$ human epidermal growth factor (PeproTech, Inc., Rocky Hill, NJ, USA), and $20 \mathrm{ng} / \mathrm{ml}$ human basic fibroblast growth factor-2 (PeproTech, Inc.). The cells were cultured at $37^{\circ} \mathrm{C}$ under $5 \% \mathrm{CO}_{2}, 95 \%$ air, and $90 \%$ humidity. The medium was replaced every third day, while primary-generated neurospheres were passaged every fifth day. Tertiary neurospheres were used in all in vitro experiments.

In vitro experimental procedures. Single-cell suspensions from tertiary neurospheres were seeded at 2,000 cells/well in 96-well plates. The treatment group (NSC+Epo) received rhEpo $(10 \mathrm{U} / \mathrm{ml})$ in the culture medium for 7 days. The control group (NSC+Vehicle) consisted of cells cultured in culture medium without rhEpo. Subsequently, cell proliferation was assessed by flow cytometry.

Neurospheres were dissociated and plated directly onto laminin-coated glass coverslips in DMEM/F12 medium containing $2 \%$ fetal bovine serum (Gibco; Thermo Fisher Scientific, Inc.), $2 \%$ B-27, and $10 \mathrm{U} / \mathrm{ml}$ rhEpo at a density of 40,000 cells/coverslip. After 7 days, immunofluorescence staining for cell differentiation analysis was performed. The cells in the control group were plated without rhEpo. The specificity of the Epo effect was investigated by applying neutralizing monoclonal antibody against Epo (mab287; $0.4 \mathrm{ng} / \mathrm{ml}$; R\&D Systems, Inc., Minneapolis, MN, USA) to the cell culture medium.

Immunofluorescence staining. We performed double immunofluorescence to determine the fate of newly differentiated cells following SCI. Briefly, 4- $\mu \mathrm{m}$ thick spinal cord paraffin-embedded sections were incubated at $4^{\circ} \mathrm{C}$ overnight with a solution containing rabbit anti-BrdU antibody $(1: 1,000$; Santa Cruz Biotechnology, Inc., Santa Cruz, CA, USA) and one of the following cell-specific mouse antibodies: $\beta$-tubulin ( $\beta$-tubulin III, 1:10,000; Santa Cruz Biotechnology, Inc.) for neurons, glial fibrillary acidic protein (GFAP, 1:10,000; Abcam, Cambridge, UK) for astrocytes, and O4 (1:10,000; R\&D Systems, Inc.) for oligodendrocytes. Subsequently, sections were incubated with tetramethyl rhodamine isothiocyanate (TRITC)-conjugated goat anti-rabbit secondary antibody (1:500; Santa Cruz Biotechnology, Inc.) and fluorescein isothiocyanate (FITC)-conjugated goat anti-mouse (1:500; Santa Cruz Biotechnology, Inc.) for $2 \mathrm{~h}$ at room temperature. The nuclei were counterstained with 4',6'-diamidino-2-phenylindole (DAPI) $(1 \mathrm{~g} / \mathrm{ml}$; Sigma-Aldrich) for $10 \mathrm{~min}$ at room temperature.

Cell immunostaining was performed as described above. The coverslips were incubated with primary antibodies ( $\beta$-tubulin, GFAP, or O4) and secondary antibody (TRITC-conjugated goat anti-rabbit or FITC-conjugated goat anti-mouse). The cell nuclei were counterstained with DAPI. The primary antibodies were replaced with normal rabbit serum in phosphate-buffered saline (PBS, 0.01 M) for the 
negative control. Both slides and coverslips were mounted, and images were acquired on a confocal microscope (Leica SP5; Leica Microsystems, Heidelberg, Germany).

Cell quantitation. For in vivo analysis, 10 sections within the SCI region were selected in each rat, and photomicrographs were analyzed using ImageJ software (National Institutes of Health, Bethesda, MD, USA). To assess NSCs proliferation, $\mathrm{BrdU}^{+} / \mathrm{DAPI}^{+}$cells were counted, and the numbers were normalized to the measured area $(512 \times 512 \mu \mathrm{m})$; this value was then presented as number $/ \mathrm{mm}^{2}$. NSCs differentiation was estimated by quantifying BrdU/ $\beta$-tubulin, BrdU/GFAP, and BrdU/O4 double-positive cells. Data were presented as a percentage relative to the total number of $\mathrm{BrdU}^{+}$cells. All data were averaged to obtain a mean number from 10 sections per rat. DAPI staining was used to determine the total number of cells.

In vitro, 10 random fields were selected in each well with three wells per group, and the numbers of DAPI-, $\beta$-tubulin-, GFAP-, and O4-positive cells were estimated. Data were presented as a percentage of the DAPI-positive cells (total cells). All cell counts were performed by observers blinded to the treatment status.

Western blot analysis. Estimation of the Epo and EpoR protein expression was performed by western blot analysis. Total lysis of the cells and spinal cords was conducted using the Radio-Immunoprecipitation Assay buffer (Thermo Fisher Scientific, Inc.), and protein concentrations were determined using the bicinchoninic acid protein assay (Thermo Fisher Scientific, Inc.). Equal amounts $(20 \mu \mathrm{g})$ of the proteins were separated by SDS-PAGE gel, which were subsequently electroblotted onto nitrocellulose membranes. The membranes were blocked with 5\% skim milk and were probed overnight at $4^{\circ} \mathrm{C}$ with the primary antibodies anti-Epo (1:800; Santa Cruz Biotechnology, Inc.) and anti-EpoR (1:800; Santa Cruz Biotechnology, Inc.). The membrane was then incubated with secondary horseradish peroxidase-conjugated antibody (1:2,000; Santa Cruz Biotechnology, Inc.) at room temperature for $2 \mathrm{~h}$. The membranes were then prepared using an electrochemiluminescence western blotting kit (Pierce, Rockford, IL, USA) according to the manufacturer's instructions. The optical density value of each band was quantified using ImageJ and was normalized to the corresponding $\beta$-actin level. Values were expressed as the fold change relative to the control value.

In vitro cell proliferation assay. NSCs proliferation was determined using flow cytometry and the number of neurospheres. Briefly, NSCs were fixed with ice-cold 70\% ethanol and washed with PBS. Subsequently, NSCs were centrifuged and resuspended in $300 \mu 1$ propidium iodide solution (Sigma-Aldrich) and $100 \mathrm{~g} / \mathrm{ml}$ RNase. Cells were then incubated at $37^{\circ} \mathrm{C}$ for $1 \mathrm{~h}$ followed by fluorescence-activated cell sorting (FACS) Calibur flow cytometer (GE, Sunnyvale, USA). Data were collected using the FACS Diva software (Becton Dickinson) and were analyzed using ModFit LT (Verity Software House, Toshan, ME, USA). The number and diameter of the neurospheres of each well were measured using an inverted phase-contrast microscope (Olympus, Hamburg, Germany) and were analyzed using ImageJ.
Evaluation of the motor neurological function. Two examiners blinded to the treatment groups evaluated the rats hindlimb motor function using the Basso, Beattie, and Bresnahan (BBB) scale score (18). Assessments were performed at 2, 8, and 14 days after SCI.

Statistical analysis. Quantitative data were first evaluated for normality with the Kolmogorov-Smirnov test. Data were presented as the mean \pm standard error of the mean (SEM), independent-samples t-tests and one-way analysis of variance (ANOVA) were used to assess significance in two-group and three-group comparisons if data were normal, otherwise nonparametric test was considered and data were presented as the median and range (P25, P75). BBB score data were evaluated with repeated measures ANOVA following rank transformation. All data analyses were performed using SPSS version 21.0 software (IBM Corp., Armonk, NY, USA). A P $<0.05$ was considered statistically significant.

\section{Results}

Epo increases NSC neurogenesis and oligodendrogenesis following SCI in vivo. Compared with the SCI+Saline group, Epo administration for 7 days significantly increased the percentage of newly generated neurons at all three time points (all $\mathrm{P}<0.01$; Fig. 1A and D), while oligodendrocytes increased on days 8 and 14 (both $\mathrm{P}<0.05$; Fig. 1B and E). Furthermore, Epo treatment resulted in fewer $\mathrm{GFAP}^{+} / \mathrm{BrdU}^{+}$immunoreactive astrocytes at all three time points compared with saline treatment (all $\mathrm{P}<0.05$ Fig. $1 \mathrm{C}$ and F).

Epo treatment increases NSC neurogenesis and oligodendrogenesis in vitro. We observed significantly more cells expressing $\beta$-tubulin $(\mathrm{P}<0.01$; Fig. $2 \mathrm{~A}$ and $\mathrm{B})$ and $\mathrm{O} 4$ $(\mathrm{P}<0.01$; Fig. $2 \mathrm{C}$ and $\mathrm{D})$ in the NSC+Epo cells compared with $\mathrm{NSC}+$ Vehicle cells. In contrast, the number of newly developed astrocytes $\left(\mathrm{GFAP}^{+} / \mathrm{BrdU}^{+}\right.$cells) decreased significantly in NSC+Epo group $(\mathrm{P}<0.05$; Fig. $2 \mathrm{E}$ and $\mathrm{F})$. These changes were not observed when we used a neutralizing monoclonal antibody against Epo, suggesting that these effects were specifically induced by Epo (Fig. 2).

Epo treatment does not affect NSC proliferation both in vivo and in vitro. We did not observe significant changes in $\mathrm{BrdU}^{+} / \mathrm{DAPI}^{+}$ cells, reflecting NSCs proliferation, at any time point following SCI between the Epo- and saline-treated rats (all $\mathrm{P}>0.05$; Fig. 3A and B). Similar results were also observed in the normal rats treated with Epo or saline (all P>0.05; Fig. $3 \mathrm{C}$ and D).

Furthermore, Epo treatment did not alter the average diameter of neurospheres ( $\mathrm{P}>0.05$; Fig. $4 \mathrm{~A}$ and $\mathrm{B}$ ) or the total number of neurospheres compared with NSC+Vehicle cells ( $\mathrm{P}>0.05$; Fig. 4A and C). Furthermore, the cell cycle analysis revealed that the proliferation index (S plus G2/M phases) was comparable between the Epo-treated cells and controls ( $\mathrm{P}>0.05$; Fig. 4D and E).

Epo treatment in vivo does not affect the proliferation or differentiation of endogenous NSCs in the normal spinal cord. At all three time points, no changes in the density of endogenous NSCs in the normal spinal cord were observed 


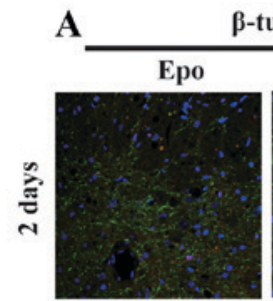

-tubulin

B

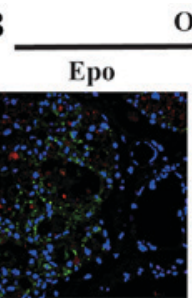

04 Saline
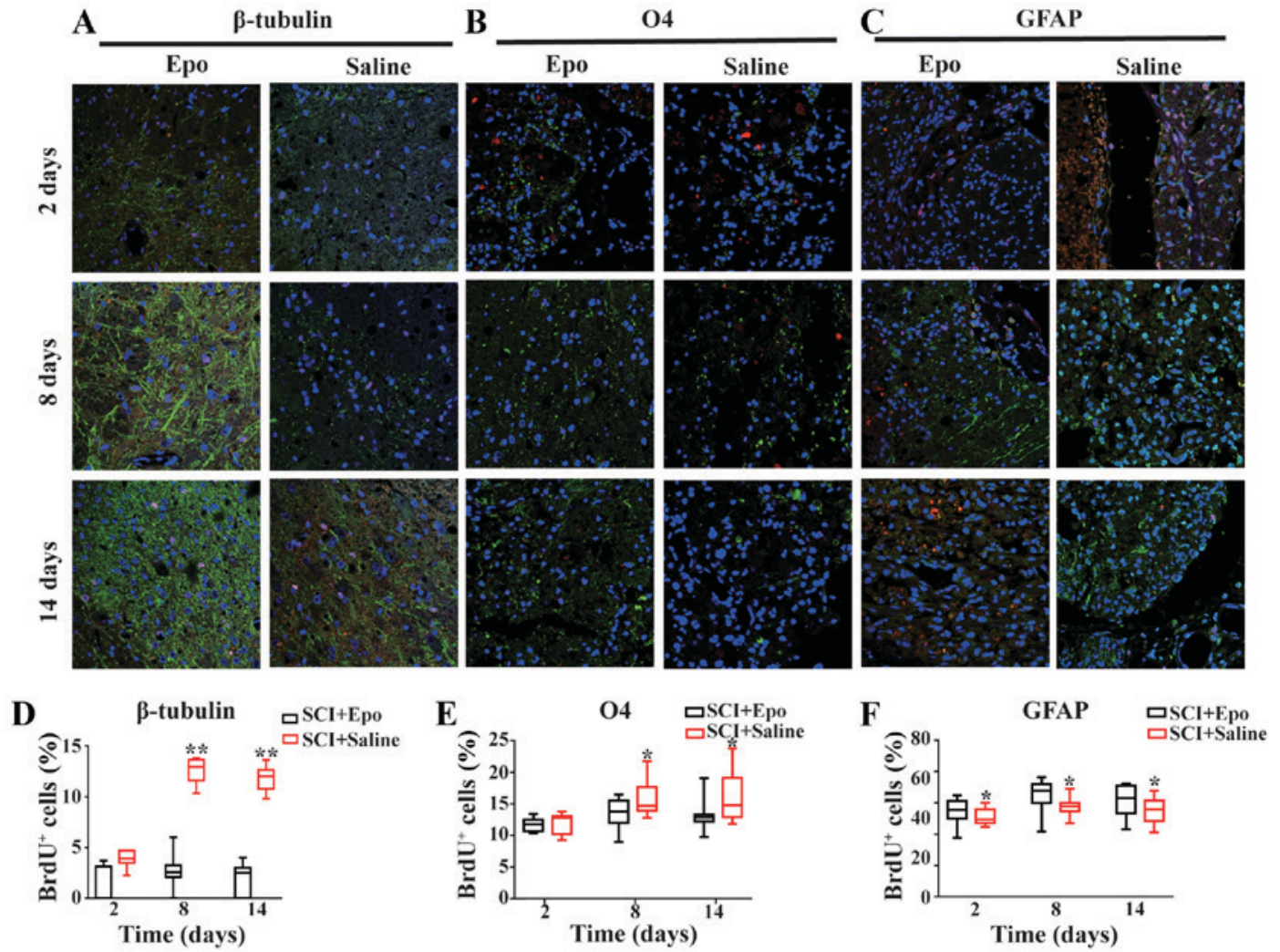

Figure 1. Effects of Epo signaling on endogenous NSC differentiation after SCI fluorescence immunostaining images (merged) showing the co-localization of (A) $\beta$-tubulin (green), (B) O4 (green), and (C) GFAP (green) with BrdU (red) at 2, 8, and 14 days in the Epo- and Saline-treated groups after SCI. DAPI (blue) was used as a nuclear counterstain. Quantitative analysis showed an increased density of (D) $\beta$-tubulin ${ }^{+} / \mathrm{BrdU}^{+}$cells at all time points and (E) $\mathrm{O} 4^{+} / \mathrm{BrdU}^{+}$cells at day 8 and day 14 with a decreased density of $(\mathrm{F}) \mathrm{GFAP}^{+} / \mathrm{BrdU}^{+}$co-labeled cells in the SCI+Epo vs. SCI+Saline groups. All data are shown as the median (range) from six animals per group in vivo. BrdU, bromodeoxyuridine; DAPI, 4',6'-diamidino-2-phenylindole; Epo, erythropoietin; GFAP, glial fibrillary acidic protein; NSC, neural stem cells; SCI, spinal cord injury; O4, oligodendrocyte marker O4. ${ }^{*} \mathrm{P}<0.05$ and ${ }^{* *} \mathrm{P}<0.01$ vs. SCI+Epo.
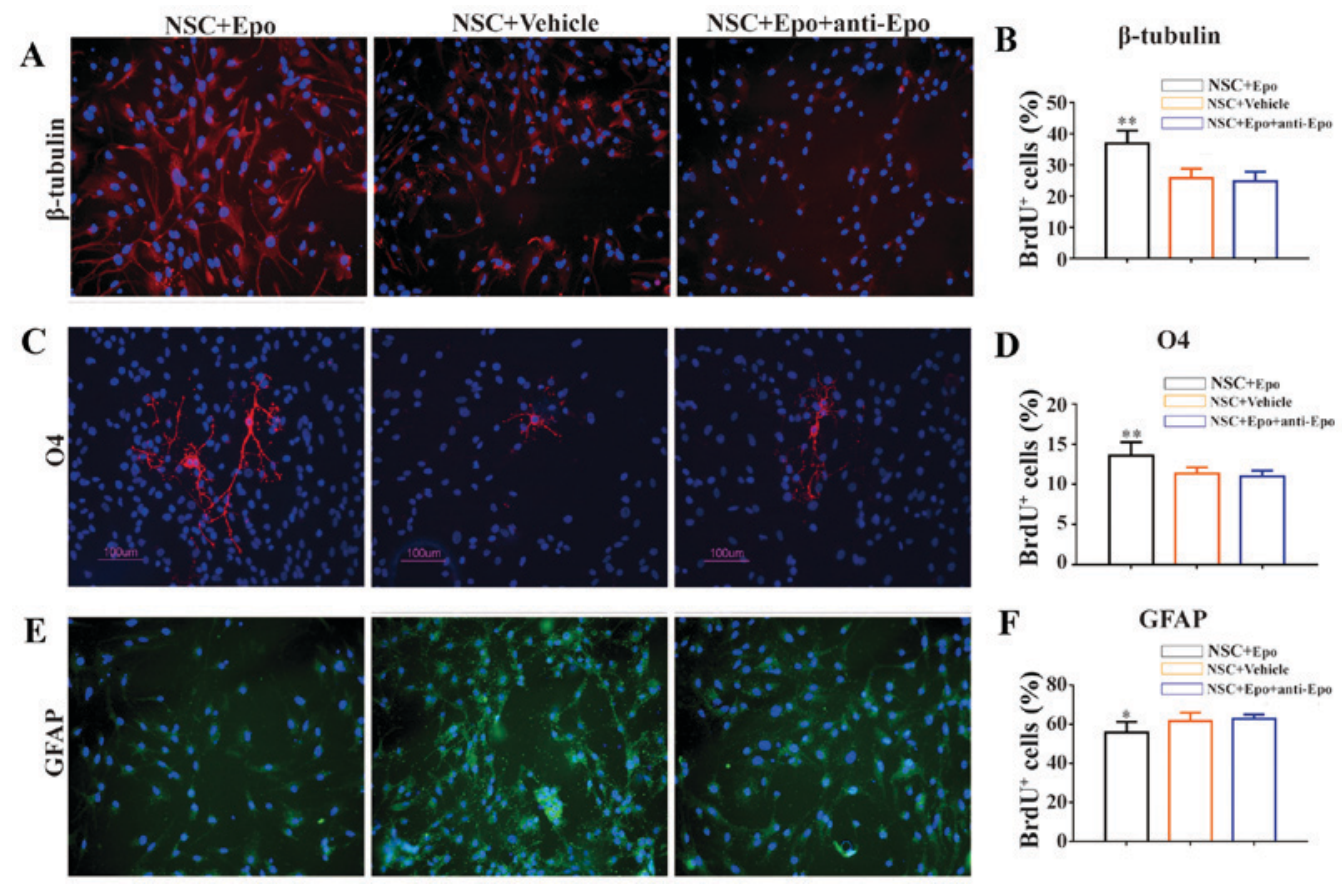

Figure 2. Effects of Epo signaling on endogenous NSC differentiation in vitro. (A) Fluorescence immunostaining images (merged) and (B) quantitative analysis showing the co-localization of $\beta$-tubulin (red). (C) Representative image (green) and (D) quantitative analysis of O4. (E) Representative image and (F) quantitative analysis of GFAP (red; E) with DAPI (blue) at day 8 following exposure to Epo, Vehicle, and Epo+anti-Epo antibody for 7 days in vitro. DAPI was used as a nuclear counterstain. Quantitative analysis showed an increased density of $\beta$-tubulin ${ }^{+} / \mathrm{BrdU}^{+}$cells and $\mathrm{O}^{+} / \mathrm{BrdU}^{+}$cells with a decreased density of $\mathrm{GFAP}^{+} / \mathrm{BrdU}^{+}$cells in the Epo vs. Vehicle groups. Antibody against Epo neutralized these effects. Scale bars, $100 \mu \mathrm{m}$. All data are shown as the mean \pm standard error of the mean from three experiments. BrdU, bromodeoxyuridine; DAPI, 4',6'-diamidino-2-phenylindole; Epo, erythropoietin; GFAP, glial fibrillary acidic protein; NSC, neural stem cells; SCI, spinal cord injury; O4, oligodendrocyte marker $\mathrm{O} 4$. ${ }^{*} \mathrm{P}<0.05$ and ${ }^{* * *} \mathrm{P}<0.01$ vs. control. 
A

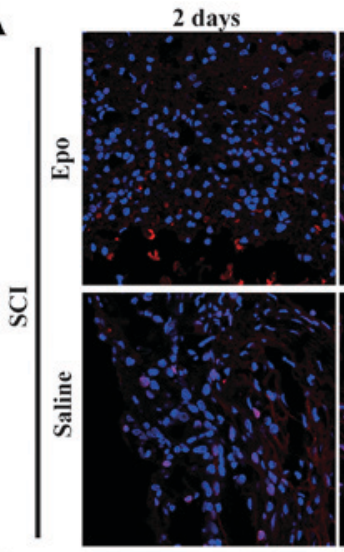

8 days

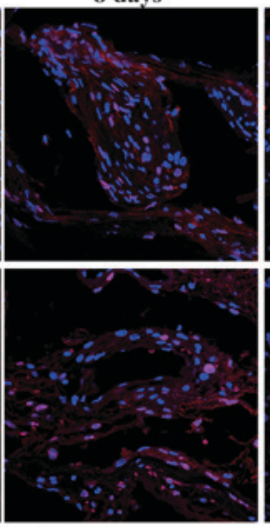

C

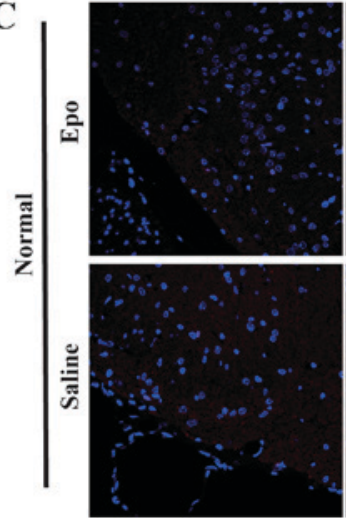

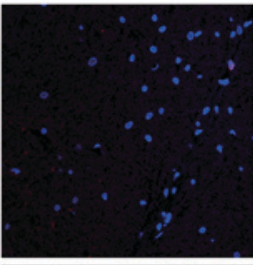

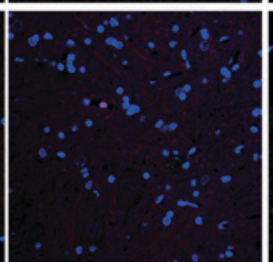

14 days
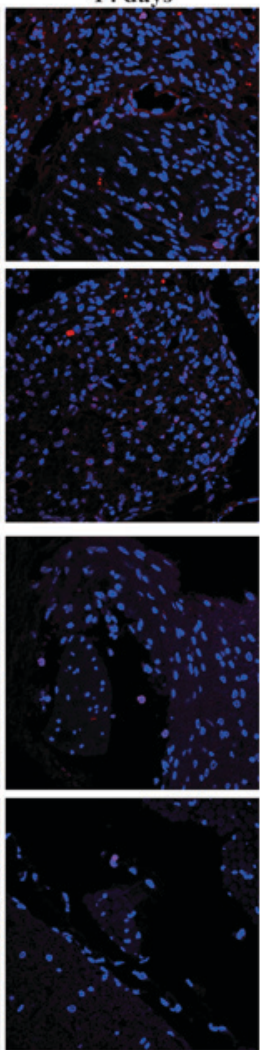

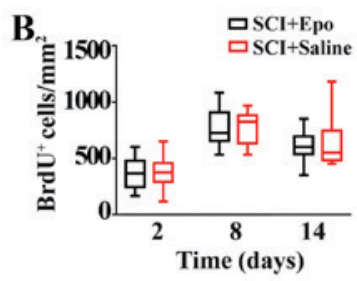

D

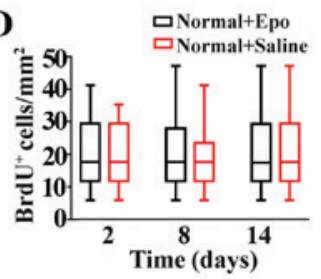

Figure 3. Effects of Epo signaling on the proliferation of endogenous NSCs derived from the adult spinal cord in vivo. (A) Fluorescence immunostaining images (merged) of BrdU (red) and DAPI (blue) at 2, 8, and 14 days in the Epo- and Saline-treated groups after SCI. (B) Quantitative analysis showed no significant difference $(\mathrm{P}>0.05)$ in the density of newly generated cells in either the Epo or Saline groups. (C) Fluorescence immunostaining images (merged) of BrdU (red) and DAPI (blue) at 2, 8, and 14 days in the Epo- and Saline-treated groups in normal rats and (D) quantitative analysis. All data are shown as the median (range) from six animals per group in vivo. BrdU, bromodeoxyuridine; DAPI, 4',6'-diamidino-2-phenylindole; Epo, erythropoietin; NSC, neural stem cells; SCI, spinal cord injury.

A

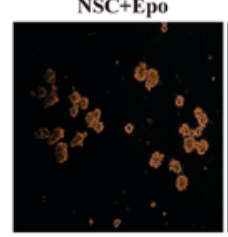

NSC+Vehicle
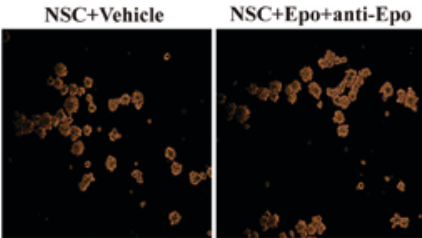

D

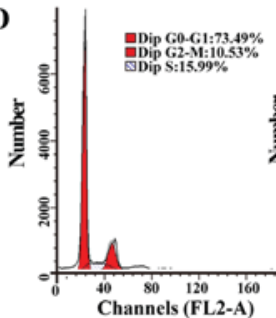

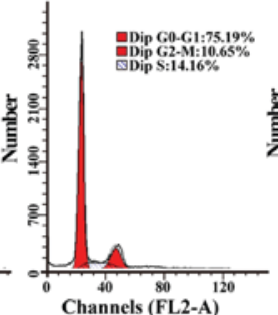

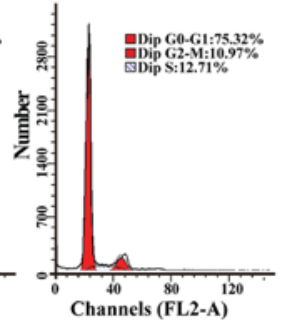

B a

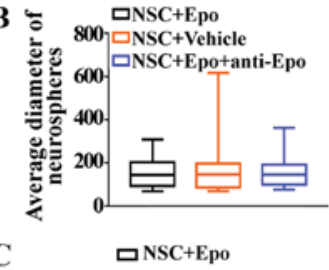

C

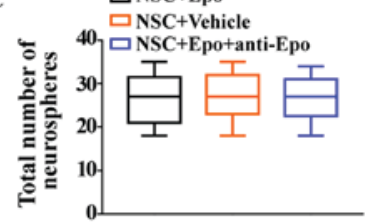

E $\overline{2}$ 口NSC+Eno

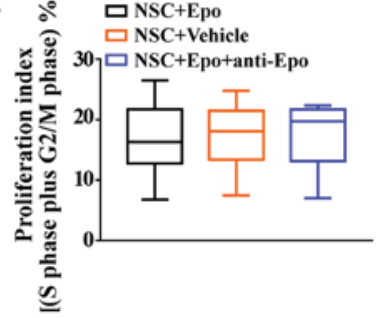

Figure 4. Effects of Epo signaling on the proliferation of endogenous NSCs derived from the adult spinal cord in vitro. Phase-contrast image of neurospheres cultured under proliferating conditions at day 8 after exposure to Epo, Vehicle or Epo+anti-Epo for 7 days in vitro (A). Histogram shows that the average diameter $(\mathrm{B})$ and total number $(\mathrm{C})$ of neurospheres were not significantly different $(\mathrm{P}>0.05)$ between any two groups. The neurospheres were disseminated into single-cell suspensions, stained with propidium iodide, and subjected to flow cytometry analysis (D); the calculated results for the proliferation index (S phase plus G2/M phase) showed that Epo signaling did not alter cell cycle progression ( $\mathrm{P}>0.05$; E). Data of total neurosphere number is shown as the mean \pm standard error of the mean, the others are shown as median (range) and all data from three experiments in vitro. Epo, erythropoietin; NSC, neural stem cells; SCI, spinal cord injury. 
A

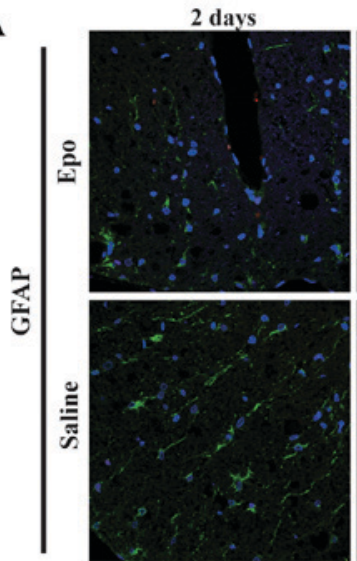

C

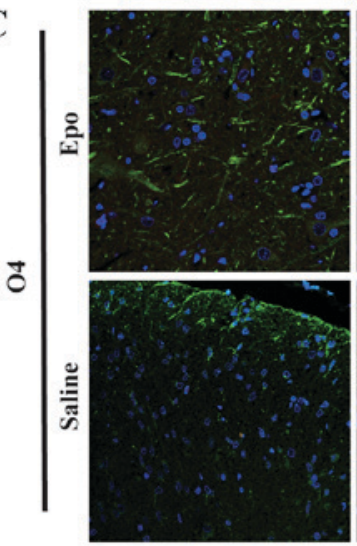

8 days
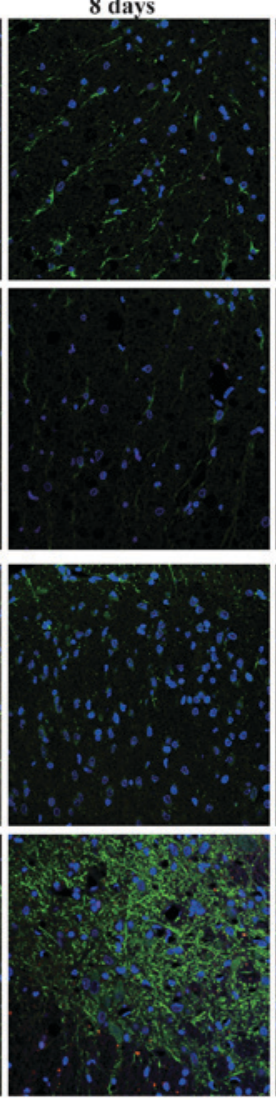

14 days

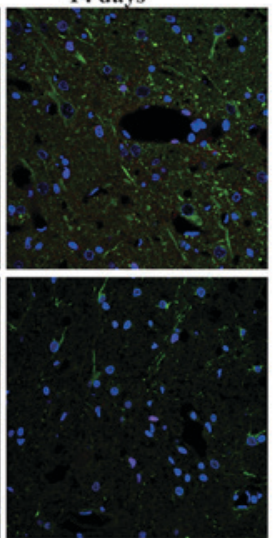

B

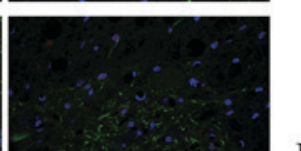

D
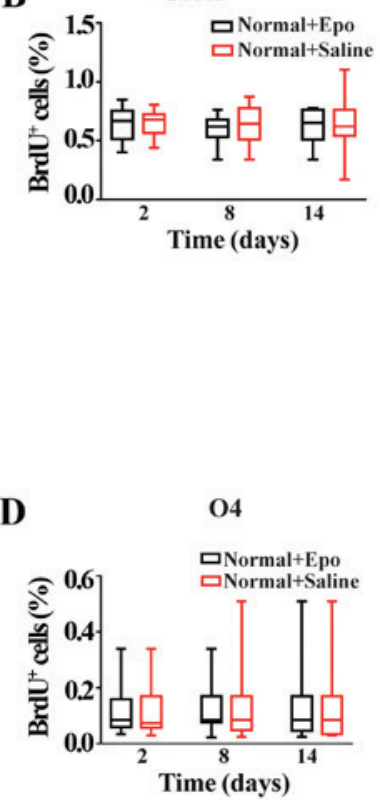

Figure 5. Effects of Epo signaling on endogenous NSC differentiation in the normal spinal cord. (A) Fluorescence immunostaining images (merged) showing the co-localization of GFAP (green), (B) Quantitative analysis showed no significant difference in $\mathrm{GFAP}^{+} / \mathrm{BrdU}^{+}(\mathrm{all} \mathrm{P}>0.05$ ). (C) Fluorescence immunostaining images (merged) showing the co-localization of O4 (green) with BrdU (red) at 2, 8, and 14 days in the Epo- and Saline-treated groups in the normal spinal cord. DAPI (blue) was used as a nuclear counterstain. (D) Quantitative analysis showed no significant difference in $\mathrm{O} 4^{+} / \mathrm{BrdU}^{+}$(all $\mathrm{P}>0.05$, D) co-labeled cells at all three time points in the Normal+Epo vs. Normal+Saline groups. All data are shown as the median (range) from six animals per group in vivo. BrdU, bromodeoxyuridine; DAPI, 4',6'-diamidino-2-phenylindole; Epo, erythropoietin; GFAP, glial fibrillary acidic protein; NSC, neural stem cells; O4, oligodendrocyte marker $\mathrm{O} 4$.

after a 7-day treatment with Epo compared with saline-treated normal rats (all $\mathrm{P}>0.05$; Fig. $3 \mathrm{C}$ and D). Moreover, we did not observe any BrdU/ $\beta$-tubulin double-positive newly generated cells in both normal spinal cord groups. Furthermore, the numbers of $\mathrm{BrdU}^{+} / \mathrm{GFAP}^{+}$and $\mathrm{BrdU}^{+} / \mathrm{O}^{+}$cells remained unchanged in both groups (all $\mathrm{P}>0.05$; Fig. 5).

Epo treatment upregulates Epo and EpoR expression both in vivo and in vitro. Compared with the low Epo and EpoR immunoreactivity in the Normal+Saline rats (Fig. 6A), these levels significantly increased in the Normal+Epo group at day $2(\mathrm{P}<0.01)$, with a maximum Epo increase of 4 -fold $(\mathrm{P}<0.01$; Fig. 6C) and a maximum EpoR increase of 2.7-fold $(\mathrm{P}<0.01$; Fig. 6D) at day 8. In contrast, Epo expression returned to normal control levels at day 14 ( $\mathrm{P}>0.05$; Fig. 6C), while the EpoR expression, which also significantly decreased at day 14, remained significantly higher than the Normal+Saline rats ( $\mathrm{P}>0.05$; Fig. 6D).

Following SCI, both Epo and EpoR immunoreactivities increased (Fig. 6A); however, there were no significant differences at days 2 or 8 ( $P>0.05$; Fig. $6 \mathrm{~F}$ and $\mathrm{G}$ ) between the $\mathrm{SCI}+$ Saline and SCI+Epo rats. Furthermore, Epo and EpoR expression decreased in both groups after day 8 , but remained significantly higher in the SCI+Epo rats at day $14(\mathrm{P}<0.01$; Fig. $6 \mathrm{~F}$ and $\mathrm{G}$ ) compared with the SCI+Saline rats.
Similar results were obtained in vitro (Fig. 6B), where a 9-fold increase in Epo $(\mathrm{P}<0.01$; Fig. $6 \mathrm{E})$ and a 5.9-fold increase in EpoR $(\mathrm{P}<0.01$; Fig. $6 \mathrm{H})$ were observed in NSC+Epo cells compared with NSC+Vehicle cells. The specificity of these effects was confirmed by the neutralizing monoclonal antibody against Epo, which resulted in no differences $(\mathrm{P}>0.05$; Fig. 6E and $\mathrm{H})$.

Epo treatment promotes motor function recovery following SCI. The initial trauma following SCI resulted in flaccid paralysis of the hindlimbs accompanied by urinary retention that was evacuated by bladder palpation twice per day. The results of BBB scores are summarized in Table I. At 2 days post-SCI, no differences were observed between the SCI+Epo and SCI+Saline rats $(\mathrm{P}>0.05)$. However, by days 8 and 14 , the treated rats scored significantly higher than the controls $(\mathrm{P}<0.05)$.

\section{Discussion}

In the present study, we investigated the effect of rhEpo treatment on neuronal recovery following SCI. Our findings provide evidence that 7 consecutive days of systemic delivery of rhEpo can activate Epo signaling and enhance not only neuronal but also oligodendrocyte differentiation following 
A

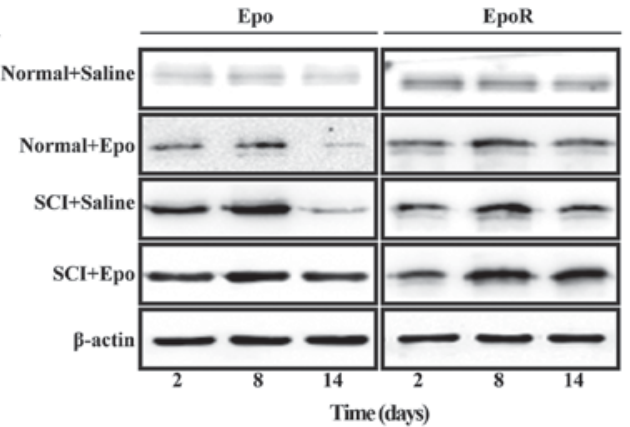

C

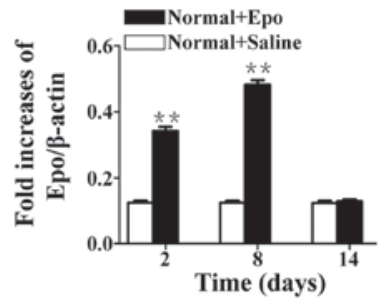

F

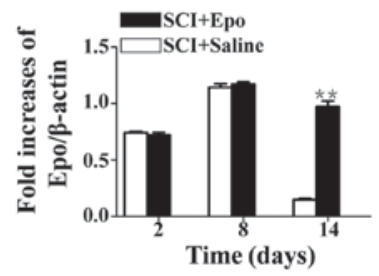

D

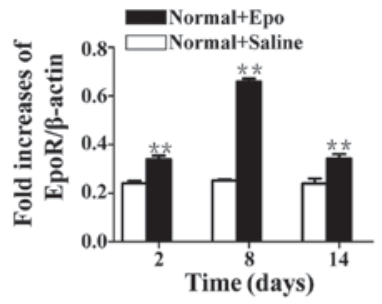

G

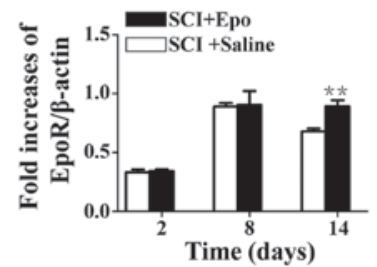

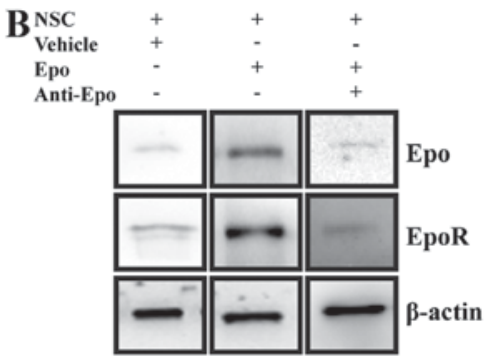

$\mathbf{E}$

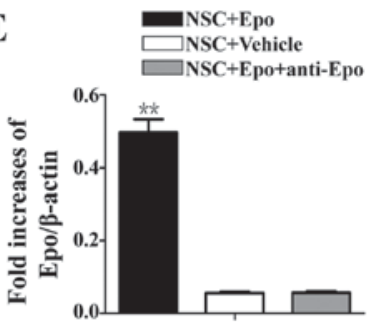

H

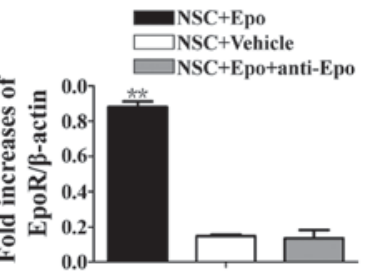

Figure 6. Levels of Epo and EpoR protein expression in vivo and in vitro. (A) Western blots of the Epo and EpoR expression following treatment with saline or Epo for 7 days in normal and SCI rats, and (B) endougenous NSCs in vitro with Epo, Vehicle or Epo+anti-Epo antibody. Western blot analysis showed the presence of two immunoreactive bands, at approximately 64 and $38 \mathrm{kDa}$, consistent with the reported molecular weights of EpoR and Epo, respectively. $\beta$-actin served as the gel-loading control. (C-H) All data were normalized to the $\beta$-actin expression, and the results are shown as the fold change relative to the control value. The data are shown as the mean \pm standard error of the mean from six animals per group in vivo or three experiments in vitro. Epo, erythropoietin; EpoR, erythropoietin receptor; NSC, neural stem cells. ${ }^{* *} \mathrm{P}<0.01$ vs. corresponding control.

Table I. Basso, Beattie and Bresnahan scores of hindlimb movements following spinal cord injury.

\begin{tabular}{lccc}
\hline & \multicolumn{3}{c}{ BBB score } \\
\cline { 2 - 4 } Groups & 2 days & 8 days & 14 days \\
\hline Epo+SCI & $0(0,1.0)$ & $6.0(4.5,7.0)^{\mathrm{a}}$ & $11.5(9.8,12.3)^{\mathrm{a}}$ \\
Saline+SCI & $0(0,0.2)$ & $2.5(1.7,3.2)$ & $6.5(5.0,8.0)$ \\
\hline
\end{tabular}

BBB scores showing the hindlimb motor function recovery level at 2, 8, and 14 days in the Epo- and Saline-treated groups following SCI. The data are shown as the median (range) from six animals per timepoint in vivo. Epo, erythropoietin; BBB, Basso, Beattie, and Bresnahan; SCI, spinal cord injury. ${ }^{\mathrm{a}} \mathrm{P}<0.01$.

SCI both in vivo and in vitro. However, no effects on proliferation or in the normal adult spinal cord were observed.

Recent studies reported a protective role of Epo and EpoR in ischemic and non-ischemic CNS injuries (1,7). Epo administration leads to substantial neuroprotection and provides a marked recovery of motor function following SCI; thus, the Epo-induced prevention of secondary injury has been proposed (10-12).
We chose the SCI contusion model because it generates highly reproducible trauma and best mimics common SCI in humans (19). The Epo dose (5,000 U/kg) was selected based on the best biochemical results obtained in preliminary studies (20). The 7-day Epo treatment regimen, which allows safe hematocrit levels, was used based on a previous study (21).

We first examined the effect of Epo signaling in the non-injured spinal cord. Ransome and Turnley (22) reported that a 7-day systemic delivery of rhEpo transiently increased the number of $\mathrm{BrdU}^{+}$cells by $30 \%$, while neurons were increased by $40 \%$ in the normal sub-granular zone of the dentate gyrus. Furthermore, a 3-week systemic administration of Epo induced $\mathrm{BrdU}^{+}$cells and CA1/CA3 neurons, in addition to an approximate $20 \%$ increase in oligodendrocytes in the healthy young mouse hippocampus (23). In contrast, our data suggest no effect of 7-day Epo treatment on the number and cell fate of endogenous NSCs of healthy spinal cords despite marked increases in Epo and EpoR expressions. Although SCI shares many pathophysiological processes with brain injury, the adult spinal cord remains a highly non-neurogenic region that is nearly completely devoid of neurogenesis under normal conditions $(12,13)$. Furthermore, adult NSCs transplanted into the neurogenic hippocampus differentiate into 
neurons, whereas the same cells transplanted into the spinal cord produce astrocytic progeny (24). However, based on our results, both Epo and EpoR expression were remarkably low in the Normal+Saline group, which is in agreement with previous studies $(4,20)$. Notably, under physiological conditions, Epo has a limited capacity to pass an intact blood-brain barrier (BBB) via a saturable transport mechanism due to its relatively high molecular weight and high degree of glycosylation structures (25). In line with this, neonates treated weekly with rhEpo either subcutaneously $(1,200 \mathrm{U} / \mathrm{kg})$ or intravenously $(1,400 \mathrm{U} / \mathrm{kg})$ to prevent transfusions did not display higher Epo levels in the cerebrospinal fluid compared with controls (26). It has been demonstrated that Epo does not cross the intact BBB of mouse (27), rat (28), and rhesus monkey (29). Furthermore, in a clinical trial, the intravenous Epo delivery within the first $6 \mathrm{~h}$ of stroke failed to exert any neuroprotective effects (30), which may be due to the fact that the $\mathrm{BBB}$ remains intact in the early hours following stroke (31).

Although Epo transport across the intact blood-spinal cord barrier (BSCB) has not been studied, we assume that Epo access to the spinal cord may be restricted. However, because a high Epo concentration is a prerequisite for its neuroprotective function (32), we postulate that Epo that is transported across the BSCB into the spinal cord contributes to an increased Epo and EpoR expression, but may be insufficient for promoting neuroprotection.

While SCI triggers endogenous NSC proliferation, most of the newly formed cells differentiate into astrocytes, and neurons are rarely found $(12,13)$. Epo signaling has been reported to enhance neuronal differentiation $(4,8,22,33)$ and production of new oligodendrocytes $(34,35)$ under both physiological and pathological conditions in the adult brain. Furthermore, Gonzalez et al (36) reported that Epo treatment promoted neurogenesis and oligodendrogliosis in neonatal rats after stroke and in healthy young mice (23). This is in line with our findings indicating a significant increase in newly generated neurons at all post-SCI time points and oligodendrocytes at days 8 and 14 in the Epo-treated SCI rats along with marked and prolonged Epo signaling activity. While local injury initiates Epo signaling, the endogenous Epo levels are insufficient for neuroprotection and repair (21). Epo has been demonstrated to increase in the spinal cord due to ruptured BSCB following SCI (25), which may be sufficient to induce a neuroprotective effect. According to our results, EpoR expression peaks on day 8 post-SCI and remains relatively high until day 14, whereas Epo remained nearly undetectable (37). Thus, we suggest that Epo administration accommodated the excessive vacant EpoR, resulting in a prolonged Epo signaling activity up until day 14 . Oligodendrocyte progenitor cells and multipotential ependymal cells account for approximately $85 \%$ of endogenous NSCs in the non-injured spinal cord. However, the rate of division of NSCs increases following SCI, resulting in the generation of new cells (19). Therefore, Epo acts most likely through promoting oligodendrogenesis rather than by protecting oligodendrocytes (37). The effect of Epo on NSC proliferation remains controversial. Indeed, previous studies reported contradictory results on proliferation by showing that Epo induced an increase $(8,22,35)$, inhibition $(23,38)$, or no effect $(35,39,40)$. Our results suggested no increase in the density of newly generated NSCs, which is similar to the effect of Epo in the hematopoietic system, i.e., driving differentiation rather than directly stimulating proliferation.

Our in vivo results were also confirmed in vitro, where we found that $10 \mathrm{U} / \mathrm{ml}$ Epo, which has a maximal effect on both neuronal production (23) and oligodendrogenesis (35), induced neuronal and oligodendrocyte differentiation to a higher level than that of the saline group with a concomitant increase in Epo and EpoR expression. Exogenous Epo directly promoted the neuronal production of NSCs derived from the brain with a 2.2-fold increase and decreased the secondary NSC sphere formation in vitro (38). Exposure to Epo and EpoR neutralizing antibodies dramatically reduced the extent of neuronal and oligodendrocyte differentiation to a level comparable to controls.

However, we observed no effect of Epo treatment on the number and diameter of neurospheres. Similarly, the percentage of cells at each cell cycle phase was not influenced by Epo. The in vitro results were also confirmed in vivo. These findings are in line with findings reported by Marfia et al (40) in which NSCs from postmortem mouse brains yielded primarily neurons (approximately 30-40\%) following the activation of Epo/EpoR signaling without altering proliferation.

Furthermore, our results suggested that Epo administration over 7 days following SCI promotes motor functional recovery, which is in line with previous reports (41).

To date, several SCI animal models have been evaluated including trauma, ischemia, inflammation, and radiationinduced models. In line with these previous studies, our results demonstrated the effectiveness of Epo treatment in promoting motor functional recovery $(9,11)$. Costa et al (41) recently reported their primary clinical trial data of Epo treatment in acute traumatic SCI vs. methylprednisolone (the current gold standard pharmacological treatment). Their results indicated a $27 \%$ absolute difference favoring Epo as an effective treatment.

Based on our present findings and on previous reports, we suggest that SCI rapidly activates the Epo signaling pathway, which could be prolonged and reinforced with exogenous Epo administration. This subsequently results in stimulating neuronal and oligodendrocyte differentiation and ultimately promoting the repair process. Scrutinizing the precise mechanisms underlying the effect of Epo signaling on neuronal repair is crucial for advancing therapeutic approaches after SCI. Epo signaling has been shown to be involved in multiple neuroprotective processes after injury, including anti-apoptotic and anti-inflammatory functions and edema reduction $(1,7,11)$. However, the precise mechanism underlying the Epo signaling pathway and its neuroprotective effects remains unknown and requires further research.

\section{Acknowledgements}

The present study was supported by the National Natural Science Foundation of China (grant no. 81171173) and the Anhui Provincial Natural Science Foundation (grant no. 11040606Q25).

\section{References}

1. Nekoui A and Blaise G: Erythropoietin and nonhematopoietic effects. Am J Med Sci 353: 76-81, 2017. 
2. Wu H, Liu X, Jaenisch R and Lodish HF: Generation of committed erythroid BFU-E and CFU-E progenitors does not require erythropoietin or the erythropoietin receptor. Cell 83: 59-67, 1995

3. Digicaylioglu M, Bichet S, Marti HH, Wenger RH, Rivas LA, Bauer C and Gassmann M: Localization of specific erythropoietin binding sites in defined areas of the mouse brain. Proc Natl Acad Sci USA 92: 3717-3720, 1995

4. Yu X, Shacka JJ, Eells JB, Suarez-Quian C, Przygodzki RM, Beleslin-Cokic B, Lin CS, Nikodem VM, Hempstead B Flanders KC, et al: Erythropoietin receptor signaling is required for normal brain development. Development 129: 505-516, 2002.

5. Kaneko N, Kako E and Sawamoto K: Enhancement of ventricular-subventricular zone-derived neurogenesis and oligodendrogenesis by erythropoietin and its derivatives. Front Cell Neurosci 7: 235, 2013.

6. McPherson RJ and Juul SE: Erythropoietin for infants with hypoxic-ischemic encephalopathy. Curr Opin Pediatr 22 139-145, 2010

7. Simon FH, Erhart P, Vcelar B, Scheuerle A, Schelzig H and Oberhuber A: Erythropoietin preconditioning improves clinical and histologic outcome in an acute spinal cord ischemia and reperfusion rabbit model. J Vasc Surg 64: 1797-1804, 2016.

8. Lu D, Mahmood A, Qu C, Goussev A, Schallert T and Chopp M: Erythropoietin enhances neurogenesis and restores spatial memory in rats after traumatic brain injury. J Neurotrauma 22 1011-1017, 2005.

9. Carelli S, Marfia G, Di Giulio AM, Ghilardi G and Gorio A Erythropoietin: Recent developments in the treatment of spinal cord injury. Neurol Res Int 2011: 453179, 2011.

10. Castaneda-Arellano R, Beas-Zarate C, Feria-Velasco AI, Bitar-Alatorre EW and Rivera-Cervantes MC: From neurogenesis to neuroprotection in the epilepsy: Signalling by erythropoietin. Front Biosci (Landmark Ed) 19: 1445-1455, 2014

11. Simon F, Scheuerle A, Gröger M, Vcelar B, McCook O, Möller P, Georgieff M, Calzia E, Radermacher $\mathrm{P}$ and Schelzig H: Comparison of carbamylated erythropoietin-FC fusion protein and recombinant humanerythropoietin during porcine aortic balloon occlusion-induced spinal cord ischemia/reperfusion injury. Intensive Care Med 37: 1525-1533, 2011.

12. Grégoire CA, Goldenstein BL, Floriddia EM, Barnabé-Heider F and Fernandes KJ: Endogenous neural stem cell responses to stroke and spinal cord injury. Glia 63: 1469-1482, 2015.

13. Johansson CB, Momma S, Clarke DL, Risling M, Lendahl U and Frisén J: Identification of a neural stem cell in the adult mammalian central nervous system. Cell 96: 25-34, 1999.

14. Qin Y, Zhang W and Yang P: Current states of endogenous stem cells in adult spinal cord. J Neurosci Res 93: 391-398, 2015.

15. Vessal M, Aycock A, Garton MT, Ciferri M and Darian-Smith C: Adult neurogenesis in primate and rodent spinal cord: comparing a cervical dorsal rhizotomy with a dorsal column transection. Eur J Neurosci 26: 2777-2794, 2007.

16. Yin ZS, Zu B, Chang J and Zhang H: Repair effect of Wnt3a protein on the contused adult rat spinal cord. Neurol Res 30: 480-486, 2008

17. Yin ZS, Zhang H, Wang W, Hua XY, Hu Y, Zhang SQ and Li GW: Wnt-3a protein promote neuronal differentiation of neural stem cells derived from adult mouse spinal cord. Neurol Res 29: 847-854, 2007.

18. Basso DM, Beattie MS and Bresnahan JC: A sensitive and reliable locomotor rating scale for open field testing in rats. J Neurotrauma 12: 1-21, 1995.

19. Sharif-Alhoseini M, Khormali M, Rezaei M, Safdarian M, Hajighadery A, Khalatbari MM, Safdarian M, Meknatkhah S, Rezvan M, Chalangari M, et al: Animal models of spinal cord injury: A systematic review. Spinal Cord 55: 714-721, 2017.

20. Zhang J, Li Y, Cui Y, Chen J, Lu M, Elias SB and Chopp M: Erythropoietin treatment improves neurological functional recovery in EAE mice. Brain Res 1034: 34-39, 2005.

21. Kaptanoglu E, Solaroglu I, Okutan O, Surucu HS, Akbiyik F and Beskonakli E: Erythropoietin exerts neuroprotection after acute spinal cord injury in rats: Effect on lipid peroxidation and early ultrastructural findings. Neurosurg Rev 27: 113-120, 2004.

22. Ransome MI and Turnley AM: Systemically delivered Erythropoietin transiently enhances adult hippocampal neurogenesis. J Neurochem 102: 1953-1965, 2007.

23. Hassouna I, Ott C, Wüstefeld L, Offen N, Neher RA, Mitkovski M, Winkler D, Sperling S, Fries L, Goebbels S, et al: Revisiting adult neurogenesis and the role of erythropoietin for neuronal and oligodendroglial differentiation in the hippocampus. Mol Psychiatry 21: 1752-1767, 2016
24. Shihabuddin LS, Horner PJ, Ray J and Gage FH: Adult spinal cord stem cells generate neurons after transplantation in the adult dentate gyrus. J Neurosci 20: 8727-8735, 2000

25. Brines ML, Ghezzi P, Keenan S, Agnello D, de Lanerolle NC, Cerami C, Itri LM and Cerami A: Erythropoietin crosses the blood-brain barrier to protect against experimental brain injury. Proc Natl Acad Sci USA 97: 10526-10531, 2000.

26. Juul SE, Harcum J, Li Y and Christensen RD: Erythropoietin is present in the cerebrospinal fluid of neonates. J Pediatr 130: 428-430, 1997.

27. Banks WA, Jumbe NL, Farrell CL, Niehoff ML and Heatherington AC: Passage of erythropoietic agents across the blood-brain barrier: a comparison of human and murine erythropoietin and the analog darbepoetin alfa. Eur J Pharmacol 505: 93-101, 2004

28. Lieutaud T, Andrews PJ, Rhodes JK and Williamson R: Characterization of the pharmacokinetics of human recombinant erythropoietin in blood and brain when administered immediately after lateral fluid percussion brain injury and its pharmacodynamic effects on IL-1beta and MIP-2 in rats. J Neurotrauma 25: 1179-1185, 2008.

29. Boado RJ, Hui EK, Lu JZ and Pardridge WM: Drug targeting of erythropoietin across the primate blood-brain barrier with an IgG molecular Trojan horse. J Pharmacol Exp Ther 333: 961-969, 2010.

30. Ehrenreich H, Weissenborn K, Prange H, Schneider D, Weimar C, Wartenberg K, Schellinger PD, Bohn M, Becker H, Wegrzyn M, et al: Recombinant human erythropoietin in the treatment of acute ischemic stroke. Stroke 40: e647-e656, 2009.

31. Latour LL, Kang DW, Ezzeddine MA, Chalela JA and Warach S: Early blood-brain barrier disruption in human focal brain ischemia. Ann Neurol 56: 468-477, 2004.

32. Castañeda-Arellano R, Feria-Velasco AI and RiveraCervantes MC: Activity increase in EpoR and Epo expression by intranasal recombinant human erythropoietin (rhEpo) administration in ischemic hippocampi of adult rats. Neurosci Lett 583: 16-20, 2014

33. Xiong Y, Mahmood A, Zhang Y, Meng Y, Zhang ZG, Qu C Sager TN and Chopp M: Effects of posttraumatic carbamylated erythropoietin therapy on reducing lesion volume and hippocampal cell loss, enhancing angiogenesis and neurogenesis, and improving functional outcome in rats following traumatic brain injury. J Neurosurg 114: 549-559, 2011

34. Zhang L, Chopp M, Zhang RL, Wang L, Zhang J, Wang Y, Toh Y, Santra M, Lu M and Zhang ZG: Erythropoietin amplifies stroke induced oligodendrogenesis in the rat. PLoS One 5: e11016, 2010

35. Jantzie LL, Miller RH and Robinson S: Erythropoietin signaling promotes oligodendrocyte development following prenatal systemic hypoxic-ischemic brain injury. Pediatr Res 74: 658-667, 2013.

36. Gonzalez FF, Larpthaveesarp A, McQuillen P, Derugin N, Wendland M, Spadafora R and Ferriero DM: Erythropoietin increases neurogenesis and oligodendrogliosis of subventricular zone precursor cells after neonatal stroke. Stroke 44: 753-758, 2013.

37. Grasso G, Sfacteria A, Passalacqua M, Morabito A, Buemi M, Macrì B, Brines ML and Tomasello F: Erythropoietin and erythropoietin receptor expression after experimental spinal cord injury encourages therapy by exogenous erythropoietin. Neurosurgery 56: 821-827, 2005.

38. Shingo T, Sorokan ST, Shimazaki T and Weiss S: Erythropoietin regulates the in vitro and in vivo production of neuronal progenitors by mammalian forebrain neural stem cells. J Neurosci 21 : 9733-9743, 2001.

39. McAdams RM, McPherson RJ, Mayock DE and Juul SE: Outcomes of extremely low birth weight infants given early high-dose erythropoietin. J Perinatol 33: 226-230, 2013.

40. Marfia G, Madaschi L, Marra F, Menarini M, Bottai D, Formenti A, Bellardita C, Di Giulio AM, Carelli S and Gorio A: Adult neural precursors isolated from post mortem brain yield mostly neurons: An erythropoietin -dependent process. Neurobiol Dis 43: 86-98, 2011.

41. Costa DD, Beghi E, Carignano P, Pagliacci C, Faccioli F, Pupillo E, Messina P, Gorio A and Redaelli T: Tolerability and efficacy of erythropoietin (EPO) treatment in traumatic spinal cord injury: A preliminary randomized comparative trial vs. methylprednisolone (MP). Neurol Sci 36: 1567-1574, 2015.

This work is licensed under a Creative Commons Attribution-NonCommercial-NoDerivatives 4.0 International (CC BY-NC-ND 4.0) License. 